\title{
Concepción de la formación de competencia en la educación superior de los ingenieros mediante la aplicación del modelo metodológico en “ $\mathbf{T}$ ”
}

\section{Conception of the training of competence in higher education of engineers through the application of the methodological model in " $T$ "}

Edilberto Antonio Llanes Cedeño

Universidad Internacional SEK, Ecuador

Gustavo Adolfo Moreno Jiménez

Universidad Internacional SEK, Ecuador

Autor para correspondencia: antonio.llanes@uisek.edu.ec

Fecha de recepción: 2 de Febrero de 2017 - Fecha de aceptación: 25 de Marzo de 2017

\section{Resumen}

En la época actual se necesita un profesional competente el cual no se puede formar bajo la concepción actual de las escuelas, porque hasta el momento se ha venido trabajando como fin aprender contenidos, lo cual en la sociedad del conocimiento es imposible por su volumen y variabilidad, por tales razones en este trabajo se tiene como objetivo: Proponer un cambio en el proceso de formación del ingeniero, mediante la incorporación al sistema de formación por competencias del modelo metodológico en $\mathrm{T}$, para la generación de capacidades generales que respondan a la sociedad y al desempeño profesional esperado en los ingenieros del siglo XXI. Se analiza el modelo de formación basado en competencias y el modelo T como planificación en aula, donde se presenta un ejemplo de integración de ambos conceptos para lograr alcanzar la competencia deseada.

Palabras claves: competencia; capacidades; modelo en t

\begin{abstract}
At the present time, a competent professional is needed, but cannot be educated under the current schools' conceptions due to its focus on the knowledge of content, what is impossible to apply on the current society with a fast variability and a big volume of information. This work propose a change in the education process of forming an engineer by incorporating the methodological approach of the model " $T$ " to help generating the general capacities that respond to society expectation and the desire performance of a 21 st century engineer. The competency-based training model and the T model as classroom planning model are analyzed and an example to integrate both concepts on a class is presented.
\end{abstract}

Key words: competition; capacities; t-mode 


\section{Introducción}

La velocidad del progreso científico, tecnológico y organizativo requiere más capacidad de respuesta, adaptabilidad y anticipación de las organizaciones, en la implementación de capacidades más cognitivas que técnicas. En este contexto, la competencia proviene de la capacidad de estar bien informado y constantemente capacitado.

Dada esta realidad, las universidades deben realizar una reformación de su sistema de formación, que permita incorporar a la sociedad un profesional con capacidades de adaptación a los cambios cada vez más rápidos en la sociedad del conocimiento.

Los sistemas educacionales precedentes al siglo XXI estaban enfocados en desarrollar un contenido determinado en el individuo, donde la materia prima eran las energías y los recursos naturales, en contraparte la sociedad de hoy denominada como "Sociedad del Conocimiento e Información“, impera el individuo y su capacidad al cambio. Por tanto, la globalización y la sociedad del conocimiento son realidades irrefutables en el mundo contemporáneo e imponen nuevos retos a las instituciones de educación donde se forman profesionales, a las que se les demandan cambios sustanciales en los procesos docentes y en los modelos para la educación. La actual sociedad requiere, formar en los futuros profesionales de la ingeniería aquellas capacidades de acción e interacción que les posibiliten desempeñarse exitosamente, con énfasis en el modo de ser que el sujeto configura y proyecta, para desenvolverse en el mundo con una visión y una identidad propia. Un profesional de la ingeniería, cuyo desempeño esté en correspondencia con las necesidades y características de la sociedad en que desempeñará sus funciones, como ciudadano responsable de las transformaciones cualitativamente superiores que habrán de producirse; un profesional que sepa hacer con conocimiento científico, que sepa extraer propuestas no sólo técnicas sino de progreso científico y humanista, un profesional flexible y trascendente, con capacidad no sólo para adaptarse a un mundo lleno de tecnología cambiante, sino para ser un promotor de cambios con una visión y una identidad propia y sobre todo que sepa auto educarse durante toda su vida.

Por lo anteriormente comentado, este trabajo tiene como objetivo: Proponer un cambio en el proceso de formación del ingeniero, mediante la incorporación al sistema de formación por competencias del modelo metodológico en T, para la generación de capacidades generales que respondan a la sociedad y al desempeño profesional esperado en los ingenieros del siglo XXI.

\section{Materiales y Métodos}

El enfoque de competencias implica cambios y transformaciones profundas en los diferentes niveles educativos, y seguir este enfoque es comprometerse con una docencia de calidad, buscando asegurar el aprendizaje de los estudiantes.

\section{Modelo de Formación basado en Competencias}

El concepto de competencia proviene del desempeño profesional en el mundo laboral, donde se define como la capacidad de resolver un problema. 
Autores como Chomsky (1970), propuso el concepto de competencia es la disposición y capacidad del individuo para lo interpretativo, por su parte Hymes (1996) plantea el concepto de competencia comunicativa como el empleo efectivo del lenguaje y de la lingüística en situaciones específicas de comunicación, teniendo en cuenta las demandas del entorno, Hernández et al. (1998) ha propuesto que las competencias "son acciones situadas que se definen en relación con determinados instrumentos mediadores", igualmente, se tienen las contribuciones de Sternberg (1997) en torno a la inteligencia práctica, la cual se refiere a la capacidad que han de tener las personas para desenvolverse con inteligencia en las situaciones de la vida. Así, muchos autores han dado su definición de competencia, sin embargo, algo común es la capacidad y el contexto.

Unos de los conceptos con mayor aceptación es el de Tobón (2005), que plantea la competencia como procesos complejos de desempeño con idoneidad en un determinado contexto y con responsabilidad (capacidad de resolver problemas cada vez más complejos de manera contextual)

Según Ruiz Iglesias (2000), los momentos o fases de una secuencia didáctica basada en competencia se sustenta en:

\section{Apertura:}

- Activación del conocimiento previo.

- Enseñanza directa o por modelamiento cognoscitivo de los procedimientos implicados en las acciones y expresadas como evidencias de aprendizaje o criterios de realización.

- Impartición del contenido

- Proceso de autorregulación.

\section{Desarrollo:}

- La práctica guiada en la fase de desarrollo

- La actividad independiente en la fase de desarrollo

- Proceso de autorregulación.

\section{Cierre:}

\section{- Autoestudio}

Se han planteado diversas críticas al enfoque de competencias en la educación. A continuación, se exponen algunas de estas críticas:

a) Se orienta la educación a lo laboral, descuidando la formación disciplinar.

El contexto laboral es muy importante para el enfoque por competencia, pues permite diseñar los planes de estudios en función de las necesidades de los sectores de la economía donde en un futuro el profesional ejercerá su trabajo. Sin embargo, existen instituciones educativas que se centran en lo laboral relegando la formación disciplinar y científica lo cual no es propio del enfoque de competencias.

b) El enfoque de competencias se centra en el hacer y descuida el ser. 
Se refiere a los programas donde los enfoques de competencia laboral han tendido a enfatizar en la ejecución de actividades y tareas, con un bajo grado de consideración de los valores y actitudes, sin considerar que lo afectivo - motivacional es decisivo en el cumplimiento de cualquier tarea con idoneidad.

c) Las competencias son lo que siempre hemos hecho.

Está relacionado con una resistencia al cambio que impide a los docentes estudiar con profundidad este enfoque y comparar sus contribuciones con lo que se ha hecho tradicionalmente en la educación.

Sin embargo, el enfoque por competencia tiene una serie de contribuciones entre las cuales se pueden destacar:

a) Énfasis en la gestión de la calidad del aprendizaje y de la docencia.

b) Formación orientada al desempeño idóneo mediante la integración del conocer, con el ser y el hacer.

c) Estructuración de los programas de formación acorde con el estudio sistemático de los requerimientos del contexto (Tobón, 2005).

d) Evaluación de los aprendizajes mediante criterios construidos en colectivo con referentes académicos y científicos.

\section{Modelo en " $\mathbf{T}$ ".}

Los modelos de las escuelas antes de la sociedad del conocimiento se centraron en el "Qué" y el "Cómo", o sea en el contenido y los medios para llegar a dominarlos, sin embargo, estaba relegado el "Para qué", que en esta sociedad es lo fundamental pues tiene implícita las capacidades y valores. Por tanto, se refiere a un cambio de paradigma, del conductista al socio cognitivo, y esta transición comienza en el aula y solo es viable desde ella.

En la sociedad del conocimiento se entiende por conocimiento: capacidades/destrezas como herramientas mentales que posibiliten el aprender y el seguir aprendiendo; contenidos, como formas de saber, sintéticos y sistémicos que posibiliten mentes bien ordenadas; métodos en forma de contenidos aplicados (el saber sin el saber hacer en una sociedad tecnológica es poco útil); y valores/actitudes si queremos que esta sociedad sea humanista. Esta nueva sociedad entiende que los contenidos y métodos son medios para desarrollar capacidades y valores (Román, 2011).

Así llegamos al modelo en " $\mathrm{T}$ ", el cual constituye un instrumento que permite la aplicación, en el aula, del paradigma socio - cognitivo - humanista. En la tabla 1, se resume los aspectos a tomar en cuenta en este modelo.

\begin{tabular}{|c|c|}
\hline \multicolumn{2}{|c|}{$\begin{array}{c}\text { Tabla } 1 \\
\text { Concepción del modelo en } \mathbf{T}\end{array}$} \\
\hline CONTENIDOS & MÉTODOS DE APRENDIZAJE \\
\hline $\begin{array}{l}\text { 1. Inteligencia como producto } \\
\text { • Contenidos que hay que aprender }\end{array}$ & 1. Inteligencia como proceso \\
\hline
\end{tabular}


- Arquitectura del conocimiento

- Organización del conocimiento en estructuras y esquemas mentales
- La inteligencia se construye a través de la utilización de estrategia de aprendizaje, utilizando métodos de aprendizaje y formas de aprendizaje científico, constructivo y significativo

$\begin{array}{ccc}\text { CAPACIDADES/DESTREZAS } & \text { OBJETIVOS } & \text { VALORES/ACTITUDES } \\ \text { 1. Inteligencia como producto } & \text { 1. Inteligencia afectiva } \\ \text { • Capacidades } & \bullet \text { Valores } \\ \bullet \text { Destrezas } & \bullet \text { Actitudes } \\ \bullet & \text { Habilidades } & \bullet \text { Micro actitudes }\end{array}$

\section{Fuente: Román (2011)}

Se denomina modelo en $\mathrm{T}$, por la manera en que se lee, de arriba hacia abajo y de izquierda a derecha; donde los contenidos y métodos de aprendizaje (se orienta al desarrollo de capacidades y no de contenidos) son medios para desarrollar capacidades y valores.

Según Román (2011), el modelo en T, es una puerta de entrada en la sociedad del conocimiento por las razones siguientes:

- Visualiza la sociedad del conocimiento y sus elementos fundamentales de una manera global y por ello actúa como mapa mental profesional del profesor (no se está viviendo una época de cambios sino un cambio de época).

- Facilita el acceso a la sociedad del conocimiento y sus códigos (al construir los métodos no se miran los contenidos del modelo en $\mathrm{T}$, sino las capacidades existentes en los mismos).

- Impulsa el cambio cultural en una organización (se trata de transitar desde la cultura del "Qué" a la cultura del "Cómo" y sobre todo a la cultura del "Para qué")

- Globaliza el pensamiento profesional y facilita su procesamiento.

- Integra adecuadamente los elementos básicos de la cultura institucional y facilita su desarrollo.

- Propicia la transición desde una escuela conductista, propia de la segunda revolución industrial (1900), a la Escuela Refundada en el marco de la sociedad del conocimiento (2000).

- Propicia el cambio del paradigma conductista, propio de la segunda revolución industrial, al paradigma socio cognitivo, propio de la revolución del conocimiento.

\section{Resultados y Discusión}

El campo tecnológico en los últimos años se ha caracterizado por un cambio continuo e impredecible en el futuro, por tal razón los ingenieros en particular deben mirar al futuro, lograr un proceso de formación que le permita aprender a aprender y poder ser competitivo en diferentes contextos.

A partir del enfoque del aprendizaje por competencias, e incorporando el modelo en $\mathrm{T}$ de planificación metodológica en el aula, se facilita la lectura del concepto de competencia, en el marco de la sociedad del conocimiento, al integrar en el mismo capacidades, valores, contenidos y métodos, para obtener un mejor desempeño profesional. 
En la tabla 2, se ejemplifica para la formación de un Ingeniero Mecánico desde el enfoque por competencia, pero aplicando el modelo $\mathrm{T}$ a la planificación del contenido a impartir en el aula para la asignatura Electrónica, la cual contribuye a la competencia de diseñar circuitos electrónicos mediante el análisis funcional de los elementos electrónicos para problemas complejos de control de maquinarias industriales y/o automotrices.

\section{Tabla 2}

\section{Modelo T para la asignatura Electrónica}

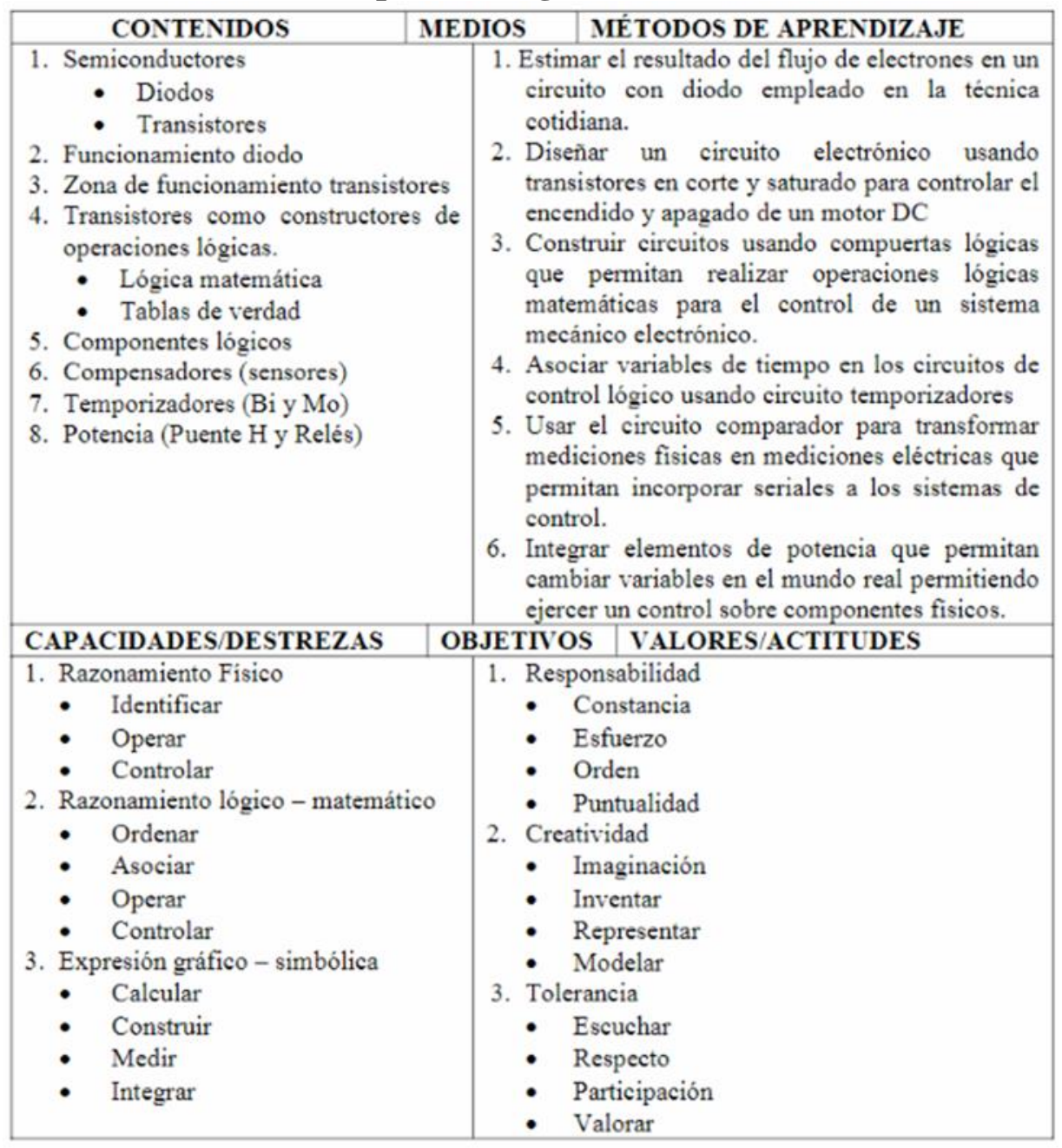

Fuente: Autores

En la anterior, mediante los métodos de aprendizaje y aprovechando los contenidos se logra crear capacidades en los estudiantes para seguir aprendiendo, cada capacidad se subdivide en destrezas y durante el desarrollo de esas capacidades se construyen valores, todo lo cual va a influir directamente en el logro de la competencia.

\section{Conclusiones}

El modelo de formación basado en competencias persigue el objetivo de formar profesionales para la sociedad del conocimiento. 
El modelo en T, es un nuevo paradigma de la planificación, que permite desde el aula, y través de los conocimientos - métodos de aprendizaje lograr la formación de capacidades y valores.

La integración del modelo de formación basado en competencias y el modelo en $\mathrm{T}$ aplicado al aula, permite desarrollar adecuadamente competencias necesarias para la nueva época.

\section{Bibliografía}

Chomsky, N. (1970). Aspectos de la teoría de la sintaxis. Madrid: Editorial Aguilar.

Hernández, C.A., Rocha, A., y Verano, L. (1998). Exámenes de Estado. Una propuesta de evaluación por competencias. Bogotá: ICFES.

Hymes, D. (1996). Acerca de la competencia comunicativa. Forma y Función, 9. Departamento de Lingüística, Universidad Nacional de Bogotá.

Román, M. (2011). Aprender a aprender en la sociedad del conocimiento. Chile: Editorial Conocimiento S.A. ISBN 956-240-435-8

Ruiz Iglesias, M. (2000). El enfoque integral del currículo para la formación de profesionales competentes. México D. F: Editorial IPN, 18.

Sternberg, R.J. (1997). Successful intelligence. New York: Simon and Shuster.

Tobón, S. (2005). Formación basada en competencia. Pensamiento complejo, diseño curricular y didáctica (2da. Ed.). Bogotá: Ecoe. 